\title{
Internet-based assessment of postoperative neurocognitive function in cardiac and thoracic aortic surgery patients
}

\author{
Suzan Uysal, PhD, ${ }^{\text {a }}$ Michael Mazzeffi, MD, ${ }^{\text {a }}$ Hung-Mo Lin, PhD, ${ }^{\text {a }}$ Gregory W. Fischer, MD, ${ }^{\text {a }}$ \\ Randall B. Griepp, MD, ${ }^{\mathrm{b}}$ David H. Adams, MD, ${ }^{\mathrm{b}}$ and David L. Reich, MD ${ }^{\mathrm{a}}$
}

\begin{abstract}
Objective: The primary purpose of this study was to determine the practicality of long-term, postoperative neurocognitive assessment via remote Internet-based testing in a cohort of patients who had undergone cardiac or thoracic aortic surgery within the previous 6 years. The secondary aim of this study was to examine the relationships among hypothermic circulatory arrest time, cardiopulmonary bypass time, and selective cerebral perfusion time with long-term postoperative neurocognitive function, as assessed by this novel testing method.
\end{abstract}

Methods: Three hundred patients who had undergone cardiac and/or proximal aortic surgery with cardiopulmonary bypass $(n=207)$, thoracic aortic surgery with hypothermic circulatory arrest $(n=67)$, or thoracic aortic surgery with hypothermic circulatory arrest and selective cerebral perfusion $(n=26)$ within the previous 6 years underwent Internet-based neurocognitive assessment.

Results: The duration of hypothermic circulatory arrest was negatively associated with processing speed scores and memory scores; arrest duration greater than 21 to 24 minutes was negatively associated with response speed scores. These associations were independent of time since surgery, age at testing, and educational level. Neither cardiopulmonary bypass duration nor selective cerebral perfusion duration was associated with test score results.

Conclusions: This study demonstrated the practicality of long-term neurocognitive assessment of patients who have undergone cardiac and thoracic aortic surgery by means of Internet-based computerized testing. Furthermore, there was a negative association between the duration of intraoperative hypothermic circulatory arrest and long-term postoperative neurocognitive function that needs further examination in prospective studies. (J Thorac Cardiovasc Surg 2011;141:777-81)

Cardiac and thoracic aortic surgical procedures are associated with a variety of adverse neurologic sequelae, such as stroke, seizure, delirium, and neurocognitive decline. Although less overt than other neurologic sequelae, postoperative neurocognitive decline may occur more commonly and may be enduring. The incidence of neurocognitive decline in patients after coronary artery bypass surgery varies widely among studies, but a pooled analysis of 448 patients in 6 comparable studies reported that $22.5 \%$ exhibited a decline relative to their preoperative performance 2 months postoperatively. ${ }^{1}$ There are, however, few studies of neurocognitive outcomes in patients after they have undergone thoracic aortic surgery, and there are no large-scale studies, partially because of the geographic distribution of patients, who are referred to centers from wide catchment areas. To overcome these difficulties in other settings, computer-

\footnotetext{
From the Departments of Anesthesiology ${ }^{\mathrm{a}}$ and Cardiothoracic Surgery, ${ }^{\mathrm{b}}$ Mount Sinai School of Medicine, New York, NY.

Disclosures: Authors have nothing to disclose with regard to commercial support.

Received for publication April 8, 2010; revisions received June 23, 2010; accepted for publication Aug 1, 2010; available ahead of print Oct 11, 2010.

Address for reprints: Suzan Uysal, PhD, Department of Anesthesiology, Box 1010,

Mount Sinai School of Medicine, 1 Gustave L. Levy Place, New York, NY

10029-6574 (E-mail: suzan.uysal@mountsinai.org).

$0022-5223 / \$ 36.00$

Copyright (c) 2011 by The American Association for Thoracic Surgery

doi:10.1016/j.jtcvs.2010.08.024
}

based neurocognitive evaluation tools have been developed that may be accessed from remote locations. The primary aim of the current investigation was to assess the practicality of long-term neurocognitive assessment using remotely accessed, Internet-based testing methodology in patients who had undergone cardiac or thoracic aortic surgery within the previous 6 years. The secondary aim of this study was to examine the relationships between hypothermic circulatory arrest time, cardiopulmonary bypass time, and selective cerebral perfusion (SCP) time and long-term postoperative neurocognitive function as assessed by this novel testing method.

\section{MATERIALS AND METHODS \\ Patient Enrollment}

The study protocol was approved by the Institutional Review Board. A total of 2234 adult patients who had undergone cardiac and/or proximal aortic surgery with cardiopulmonary bypass (CPB) or thoracic aortic surgery with hypothermic circulatory arrest (HCA) as performed by 1 of 4 surgeons within the 5-year period (July 1, 2002, to July 1, 2007) preceding initiation of this study were identified; 2234 letters were mailed, inviting patients to participate in the study. Of those patients, $714(32 \%)$ responded by telephone or reply card; 443 indicated interest in participating in the study. An investigator then spoke with those patients and obtained informed consent by telephone or in written form. For this pilot investigation, recruitment ended once 300 patients had undergone neurocognitive testing. 


\author{
Abbreviations and Acronyms \\ $\mathrm{CPB}=$ cardiopulmonary bypass \\ CSI $=$ Cognitive Stability Index \\ $\mathrm{HCA}=$ hypothermic circulatory arrest \\ $\mathrm{SCP}=$ selective cerebral perfusion
}

\section{Neurocognitive Testing}

Computerized neurocognitive testing was performed using the Cognitive Stability Index (CSI) HeadMinder battery (HeadMinder, Inc., New York, NY) from Windows (Microsoft, Redmond, Wash) Internet Explorer web browsers. Study subjects took the test at their home computers or at the study's institution. Patients were assigned a study identification number by which they accessed a password-protected website. The data were encrypted using the Secure Sockets Layer protocol and stored on a server using methods compliant with federal and New York State privacy regulations and Health on the Net guidelines.

Once subjects accessed the HeadMinder website, they provided information regarding their familiarity with computers and keyboards by rating their use of 7 technologies (E-mail, Internet, computer keyboard, computer mouse, ATM, calculator, and television remote control) on a 3 -point Likert scale ( $1=$ never used; $2=$ ever used; 3 = used within the past month). These scores were summed to create a Technology Usage Score ranging from 0 to 14 , with higher scores indicating greater proficiency.

The CSI battery consists of 10 subtests that are designed to minimize the effects of intersubject differences in computer experience and language skills by requiring use of only the spacebar and the number and backspace keys and by using nonverbal stimuli. Task instructions are presented before each subtest. Each subtest has an error check that returns the test taker to the instructions if the system detects that the test taker has not understood the instructions. Built-in auto-invalidation criteria flag performances that are likely to have been confounded by extraneous factors, such as distractedness and poor effort. The CSI subtests are scored according to the number of correct responses and response latencies.

The 10 CSI subtests resolve into 4 neurocognitive factors: Response Speed, Processing Speed, Memory, and Attention. The CSI subtests and methods used to derive the factor scores have been described previously. $^{2}$ The Response Speed factor reflects motor speed; the subtest scores that contribute to this factor's scores are the latencies to respond to simple reaction-time tasks. The Processing Speed factor reflects cognitive processing speed; the subtest scores that contribute to this factor's scores are the latencies to respond on visual search and decoding tasks. The Memory factor reflects the ability to recall recent information; the subtest scores that contribute to this factor's scores are the number of responses in which the subject correctly identifies the screen location of 9 items under both learning and delayed-recall conditions. The Attention factor reflects attention capacity (ie, the number of bits of information that can be "held on line" at a given time) and working memory (ie, the ability to hold information in mind and mentally manipulate it).

The CSI factor scores have proven to have moderate test-retest reliabilities in a sample of 284 healthy subjects (Response Speed, $r=.80$; Processing Speed, $r=.78$; Attention, $r=.73$; Memory/Learning, $r=.68$ ). Concurrent validity of the CSI factors with standard neuropsychological test measures has been established (ie, Trail Making Test, Symbol Digit Modalities Test, Buschke Selective Reminding Test, and Symbol Search and Digit Span subtests of the Wechsler Adult Intelligence Scale-III). Clinical diagnostic validity of the CSI has been established in clinical samples, including mild-moderate traumatic brain injury, adult attention deficit/ hyperactivity disorder, and mild Alzheimer disease. ${ }^{2}$

\section{Statistical Methods}

For each subject, the 4 factor scores were transformed to z-scores by HeadMinder by referencing to an age-matched normative sample. ${ }^{3}$ All descriptive and statistical analyses were performed by a coauthor academic biostatistician at the authors' institution (H.L.). The study cohort was divided into 3 groups based on perfusion and brain-protection methods used during surgery: those who underwent cardiac and/or proximal aortic procedures with CPB only (CPB group); those who underwent thoracic aortic procedures with CPB and at least 1 period of HCA (HCA group); and those who underwent thoracic aortic procedures with $\mathrm{CPB}$ and periods of both HCA and SCP (SCP group). For group comparisons, chi-square tests were used for binary categorical variables (gender, remote testing, and intraoperative aprotonin), Student $t$-tests or analysis of variance (ANOVA), allowing heterogeneous variance across the groups, were used for normally distributed continuous variables (neurocognitive factor scores, age, education level, Technology Usage Score, and time since surgery). Nonparametric Kruskal-Wallis tests were used for non-normally distributed continuous CPB times, HCA times, and SCP times.

Multiple linear regression analysis was used to model the associations among CPB time (exclusive of HCA and SCP time), HCA time (exclusive of SCP time), and SCP time to each of the neurocognitive factor scores. The models were adjusted for group status (CPB, HCA, or SCP); intraoperative use of aprotinin; time since surgery; age at testing; educational level (high school or lower, college, or postgraduate); gender; Technology Usage Score; and whether remote computer testing was conducted. To explore the possibility of nonlinear relationships among CPB, HCA, and SCP times and postoperative neurocognitive performance, quadratic polynomial terms for $\mathrm{CPB}$, HCA, and SCP times were tested for statistical significance. If detected, piecewise regression analysis was performed to confirm the association. ${ }^{3}$ Two-way interactions among the 3 perfusion/ brain protection times were also tested to examine for synergistic effects. Interactions among group indicators and the three perfusion/brain protection times were also tested to assess whether the time effects differed across groups. The level of statistical significance was set to be 0.05 . All statistical analyses were carried out using SAS 9.1.3 (SAS Institute, Inc., Cary, NC).

\section{RESULTS}

A total of 300 patients underwent neurocognitive testing: 207 in the CPB group, 67 in the HCA group, and 26 in the SCP group. All testing was completed during a 1-year period, between 0.5 and 5.8 years since surgery. The CPB group consisted of patients who underwent coronary artery bypass grafting procedures, valve procedures, coronary artery bypass grafting procedures and valve procedures, or ascending aortic procedures without HCA or SCP. The HCA group consisted of patients who underwent ascending aortic procedures or "hemi-arch" repairs, in which relatively brief periods of HCA were planned. The SCP group consisted of patients who underwent aortic arch procedures. Other characteristics of the three groups are presented in Table 1. There were no differences among the groups in gender proportion, level of education, percentage of subjects taking the test remotely as opposed to taking it at the hospital, Technology Usage Score, incidence of hypertension at time of surgery, or incidence of diabetes at time of surgery. The groups differed in age at the time of testing, time from surgery to testing, intraoperative use of aprotinin, CPB duration, and HCA duration. 
TABLE 1. Group characteristics

\begin{tabular}{|c|c|c|c|c|}
\hline Variable & CPB $(n=207)$ & $\operatorname{HCA}(n=67)$ & $\operatorname{SCP}(n=26)$ & $P$ value \\
\hline Gender $(\%$ male $)$ & 67.7 & 73.1 & 76.9 & $.49 *$ \\
\hline Age $(y)($ mean \pm SD) & $66.2 \pm 12.9$ & $63.3 \pm 16.3$ & $57.9 \pm 13.6$ & $.01 \dagger^{\prime} \|$ \\
\hline Education $(y)($ mean $\pm \mathrm{SD})$ & $14.8 \pm 3.51$ & $14.5 \pm 3.45$ & $14.5 \pm 3.1$ & $.82 \dagger$ \\
\hline Technology usage score (mean \pm SD) & $10.4 \pm 3.3$ & $10.7 \pm 3.2$ & $10.1 \pm 3.6$ & $.69 \dagger$ \\
\hline Remote testing (percent) & 83.6 & 80.6 & 76.9 & $.64^{*}$ \\
\hline Time since surgery $(y)($ mean $\pm \mathrm{SD})$ & $2.1 \pm 1.1$ & $3.1 \pm 1.3$ & $2.6 \pm 1.1$ & $<.001 \dagger^{\prime} \S^{\prime} \|$ \\
\hline Intraoperative aprotinin (percent) & 24.2 & 52.2 & 76.9 & $<.001 *, \varsigma$ \\
\hline Hypertension (percent) & 77 & 77 & 92 & $.19^{*}$ \\
\hline Diabetes (percent) & 12 & 3 & 4 & $.07 *$ \\
\hline CPB time (h) (median, range) & $2.6(0.6-6.5)$ & $3.8(1.6-6.6)$ & $2.2(1.4-7.6)$ & $<.001 \S^{\prime} \|$ \\
\hline HCA time (h) (median, range) & - & $0.3(0.2-0.8)$ & $0.5(0.2-0.7)$ & $.001 \ddagger$ \\
\hline$\underline{\text { SCP time }(\mathrm{h}) \text { (median, range) }}$ & - & - & $0.8(0.1-1.6)$ & \\
\hline
\end{tabular}

$* P$-value obtained based on chi-square test. $\dagger$ ANOVA. $\ddagger$ Wilcoxon/Kruskal-Wallis Test. $\S$ The CPB group differed significantly from the HCA group $(P<.05)$. $\|$ The CPB group differed significantly from the SCP group $(P<.05)$. ๆThe HCA group differed significantly from the SCP group $(P<.05)$.

Table 2 presents the mean neurocognitive factor $\mathrm{z}$-scores for the three groups. Some test scores were flagged as invalid, so the number of subjects with test data in each group is fewer than the number who completed testing. There were no significant differences among the three groups in any of the 4 factor scores. There was a trend toward worse Response Speed scores in the SCP group (average $\mathrm{z}=-1.35$ for the SCP group compared to -0.92 for the CPB group and -0.62 for the HCA group; $P=.06$ ).

Table 3 presents the results of 4 multivariate regression analyses examining the independent effects of CPB duration, HCA duration, and SCP duration on the neurocognitive factor z-scores, controlling for aprotinin use, time since surgery, gender, age at testing, level of education, Technology Usage Score, and remote testing status. The beta coefficients represent the estimated average difference in the factor $\mathrm{z}$-scores given a unit change in the independent variable; the sign indicates whether the relationship is positive or negative. None of the two-way interactions among the 3 perfusion/brain protection times was significant, suggesting their independent association with the cognitive outcomes. In addition, interactions between group indicators and the 3 perfusion/brain protection times were all nonsignificant, suggesting that the effects of perfusion/brain protection times did not differ across groups.

The durations of CPB and SCP were unrelated to any of the 4 factor z-scores. The duration of HCA, however, was negatively associated with Processing Speed z-scores $(P<.01)$ and Memory z-scores $(P<.01)$. Specifically, for

TABLE 2. Mean (SD) neurocognitive factor z-scores by group

\begin{tabular}{lcccc}
\hline & $\begin{array}{c}\text { CPB } \\
(\mathbf{n}=\mathbf{1 7 1})\end{array}$ & $\begin{array}{c}\text { HCA } \\
(\mathbf{n}=\mathbf{5 8})\end{array}$ & $\begin{array}{c}\text { SCP } \\
(\mathbf{n}=\mathbf{2 3})\end{array}$ & $\begin{array}{c}\boldsymbol{P} \\
\text { value* }^{*}\end{array}$ \\
\hline Response speed & $-0.92 \pm 1.26$ & $-0.62 \pm 1.15$ & $-1.35 \pm 1.30$ & .06 \\
Processing speed & $-1.29 \pm 1.04$ & $-1.04 \pm 1.15$ & $-0.96 \pm 1.15$ & .25 \\
Attention & $-0.45 \pm 1.28$ & $-0.27 \pm 1.22$ & $-0.78 \pm 1.14$ & .21 \\
Memory & $-0.89 \pm 1.35$ & $-0.59 \pm 1.25$ & $-0.72 \pm 1.32$ & .33 \\
\hline
\end{tabular}

$* P$ value obtained based on ANOVA. every 10-minute increase in exposure to HCA, the Processing Speed and Memory z-scores were reduced by 0.64 and 0.70 , respectively. Attention z-scores also demonstrated a similar trend that did not reach statistical significance (ie, for any 10-minute increase in exposure to HCA, the change in z-score was $-0.36 ; P=.09$ ). HCA time was not significantly associated with Response Speed z-scores $(P=.67)$.

We explored the possibility of a nonlinear relationship among CPB, HCA, and SCP times and neurocognitive performance. There was a marginally significant association between HCA duration and Response Speed, as indicated by a quadratic polynomial term of HCA time $(P=.07$; Table 3). The negative quadratic polynomial term indicates that the relationship between HCA time and Response Speed score changed from positive to negative as HCA time increased. The inflection point of the curve was estimated to be 24 minutes. As a confirmatory test, we performed a piecewise regression to estimate the inflection point and slopes of the curve before and after the inflection point. By this method, the inflection point was at $21.5 \mathrm{~min}-$ utes; the slope before the inflection point was $4.24(95 \% \mathrm{CI}$, -5.76 to +14.23$)$; and the slope after the inflection point was -4.86 (95\% CI, -8.91 to -0.76$)$. Thus, HCA durations greater than 21 to 24 minutes were negatively associated with Response Speed z-scores. Specifically, after $24 \mathrm{~min}$ utes of HCA time, for any 10-minute increase, the Response Speed z-score was reduced by 0.81 .

With regard to surgical variables, the multivariate regression analysis indicated that aprotinin use and time since surgery were not significantly associated with any of the factor $\mathrm{z}$-scores (although there was a trend of annual improvement of Processing Speed score of 0.1 for time since surgery; $P=.06)$. With regard to demographic variables, age was negatively associated with all 4 factor z-scores; level of education was positively associated with Processing Speed and Attention factor z-scores; and gender was not associated with any factor z-scores. With regard to computer 
TABLE 3. Multiple regression analyses of the relationships of CPB, HCA, and SCP times to 4 neurocognitive test scores, adjusting for potential confounders

\begin{tabular}{|c|c|c|c|c|c|c|c|c|c|c|c|c|}
\hline \multirow[b]{2}{*}{ Effect } & \multicolumn{3}{|c|}{ Response speed } & \multicolumn{3}{|c|}{ Processing speed } & \multicolumn{3}{|c|}{ Attention } & \multicolumn{3}{|c|}{ Memory } \\
\hline & Beta* & Std Err* & $P$ value & Beta & Std Err & $P$ value & Beta & Std Err & $P$ value & Beta & Std Err & $P$ value \\
\hline Intercept & -1.04 & 0.57 & .07 & -0.85 & 0.52 & .11 & -0.87 & 0.56 & .12 & -0.92 & 0.67 & .17 \\
\hline HCA group indicator & -2.39 & 1.68 & .16 & 1.17 & 0.44 & $<.01$ & 0.88 & 0.49 & .07 & 1.68 & 0.60 & $<.01$ \\
\hline SCP group indicator & -2.44 & 1.71 & .15 & 2.40 & 0.76 & $<.01$ & 1.10 & 0.83 & .18 & 1.91 & 1.05 & .07 \\
\hline Total CPB time (h) (all patients) & -0.04 & 0.07 & .58 & 0.09 & 0.06 & .12 & 0.06 & 0.06 & .34 & 0.04 & 0.07 & .60 \\
\hline Total HCA time $(\mathrm{h})(\mathrm{HCA}+\mathrm{SCP})$ & 14.21 & 8.27 & .09 & -3.82 & 1.17 & $<.01$ & -2.14 & 1.25 & .09 & -4.17 & 1.52 & $<.01$ \\
\hline Quadratic Polynomial term (h) $\dagger$ & -17.66 & 9.73 & .07 & & & & & & & & & \\
\hline Total SCP time (h) (SCP) & -0.68 & 0.65 & .29 & -0.46 & 0.49 & .35 & -0.37 & 0.57 & .52 & 0.42 & 0.73 & .57 \\
\hline Intraoperative aprotinin & -0.10 & 0.17 & .57 & -0.04 & 0.14 & .77 & -0.02 & 0.16 & .91 & -0.16 & 0.19 & .39 \\
\hline Time since surgery $(\mathrm{y})$ & -0.01 & 0.06 & .88 & 0.10 & 0.05 & .06 & -0.01 & 0.06 & .88 & 0.03 & 0.07 & .69 \\
\hline Male gender & 0.24 & 0.16 & .12 & 0.09 & 0.13 & .51 & -0.03 & 0.16 & .86 & -0.25 & 0.17 & .15 \\
\hline Age (y) & -0.02 & 0.01 & $<.01$ & -0.03 & 0.00 & $<.01$ & -0.02 & 0.01 & $<.01$ & -0.02 & 0.01 & $<.01$ \\
\hline College degree & 0.25 & 0.19 & .20 & 0.32 & 0.17 & .06 & 0.44 & 0.19 & .02 & 0.01 & 0.21 & .97 \\
\hline Postgraduate degree & 0.10 & 0.21 & .63 & 0.36 & 0.18 & .04 & 0.52 & 0.20 & $<.01$ & -0.03 & 0.24 & .91 \\
\hline Remote testing & 0.39 & 0.22 & .07 & 0.75 & 0.22 & $<.01$ & 0.78 & 0.21 & $<.01$ & 0.71 & 0.25 & $<.01$ \\
\hline Technology usage score & 0.10 & 0.03 & $<.01$ & 0.02 & 0.02 & .33 & 0.05 & 0.03 & .04 & 0.06 & 0.03 & .07 \\
\hline
\end{tabular}

All 2-way interactions between the 3 types of perfusion/brain protection time were nonsignificant. All interactions between CPB or HCA time with group indicators were nonsignificant. *Beta coefficient and its standard error from the linear regression model. $\dagger$ Estimated inflection point for HCA is $14.21 / 2 / 17.66=0.40 \mathrm{hr}(=24 \mathrm{~min})$.

experience and access, Technology Usage Scores were positively correlated with Processing Speed and Attention scores, whereas remote testing status was positively correlated with Processing Speed, Attention, and Memory scores.

\section{DISCUSSION}

This study examined postoperative neurocognitive function in a cohort of 300 patients who had undergone cardiac or thoracic aortic surgery within the previous 6 years at a single center. We used novel testing technology to assess 4 neurocognitive factors: Response Speed, Processing Speed, Attention, and Memory. Perfusion technology classification (CPB alone; $\mathrm{CPB}$ with $\mathrm{HCA}$; or $\mathrm{CPB}$ with $\mathrm{HCA}$ and $\mathrm{SCP}$ ) was not associated with intergroup differences in test scores, although there was a trend toward worse Response Speed scores in the SCP group. Multivariate analysis revealed that HCA duration was negatively associated with Processing Speed and Memory scores. Furthermore, a marginally significant trend in the data suggested a possible negative relationship between HCA duration and Attention scores. Beyond 21 to 24 minutes of HCA, there was a statistically significant negative association between HCA duration and Response Speed scores; overall there was a nonsignificant trend toward a nonlinear relationship between HCA duration and Response Speed. In contrast, neither CPB duration nor SCP duration was associated with neurocognitive test scores.

Our finding of an inverse relationship between HCA duration and postoperative neurocognitive test scores is consistent with the findings of several prospective studies that examined pre- to postoperative change in neurocognitive function. In an earlier prospective study of 77 patients who had undergone cardiac and thoracic aorta surgery and had been classified as having no HCA, 1 to 24 minutes of HCA, or $\geq 25$ min of HCA, we found that the group with HCA times $\geq 25$ minutes had greater declines in measures of memory and fine motor function relative to the other 2 groups at their postoperative assessments (median $=54$ days). ${ }^{4}$ Svensson and colleagues found that in 30 patients who underwent aortic arch surgery, circulatory arrest times correlated inversely with 7 of 51 neurocognitive outcome measures 6 months postoperatively. ${ }^{5}$

Our finding that duration of $\mathrm{CPB}$ was unrelated to any of the neurocognitive factor scores is consistent with the majority of prospective studies in patients undergoing cardiac and thoracic aortic surgery. In a study of 240 patients who had undergone coronary artery bypass surgery and in another study of 30 patients who had undergone cardiac valvular surgery, the duration of CPB was not a predictor of postoperative neurocognitive decline. ${ }^{6,7}$ The previously cited study by Svensson and colleagues of 30 patients undergoing aortic arch repairs found no relationship between CPB duration and neurocognitive test performance 6 months postoperatively. ${ }^{5}$

Our sample of patients who had undergone procedures with SCP was very small $(n=26)$, so our findings of a trend toward worse Response Speed scores in the SCP group and the lack of association between SCP duration and neurocognitive test scores must be interpreted in this light. Furthermore, we cannot evaluate these findings in the broader context because the literature addressing SCP and neurocognitive outcomes is limited. A prospective study of 42 patients who had undergone aortic arch surgery and had 
been randomly assigned to either SCP or HCA found no significant difference between the 2 groups in incidence of cognitive decline at either 6 or 12 weeks postoperatively. ${ }^{8}$ Another prospective study of neurocognitive function in 22 patients who had undergone aortic arch repairs with SCP found no decline at 7 days or 2 months postoperatively. ${ }^{9}$

The major aim of this study was to determine the practicality of long-term, postoperative neurocognitive assessment via remote Internet-based testing in patients who had undergone cardiac and thoracic aortic surgery. Centers performing complex cardiac and thoracic aortic surgery draw patients from large geographic areas, so their ability to perform long-term postoperative assessments by traditional paper-and-pencil testing is limited for practical reasons. More rigorous assessment of the neurocognitive outcomes of complex cardiac and thoracic aortic surgery could be facilitated by remote testing modalities, as demonstrated by this study.

The main limitation of this study was that neurocognitive testing was performed postoperatively only; this obviated the evaluation of changes in neurocognitive function. Patients who required longer durations of HCA may have had poorer neurocognitive function preoperatively or may have had poorer postoperative neurocognitive function secondary to occult cerebrovascular disease or cardiac failure. We agree with the Statement of Consensus on Assessment of Neurobehavioral Outcomes After Cardiac Surgery published in 1995 that "the individual change in performance from baseline to a time after operation is essential to any evaluation of the impact of the operation or any intervention." ${ }^{10}$ The findings of this pilot investigation are highly preliminary, but they demonstrate the practicality of using these methods for further investigations into the evaluation of pre- to postoperative changes in neurocognitive function.

Another limitation of this study is the selection biases that are inherent in postoperative surveys; use of Internet-based technology to assess cognitive function may also result in selection bias because patients of lower socioeconomic status may be undersampled.

Despite these limitations, the current investigation is the largest report of neurocognitive outcome in long-term survivors of thoracic aortic surgery, and it includes a large comparison group of survivors of coronary artery bypass surgery and valvular cardiac surgery. The authors have been engaged in longitudinal evaluation of the neurocognitive outcomes of thoracic aortic surgery for more than 10 years and have not previously been able to investigate large numbers of patients because of the lack of preoperative hospital admission days and the high attrition rates related to the large geographic distribution of patients' domiciles. The authors conducted this investigation mainly to demonstrate the practicality of remote computerized neurocognitive assessment for future, prospective longitudinal studies. Such prospective studies should include further evaluation of the sensitivity, reliability, and validity of remote neurocognitive testing tools.

This study demonstrated that long-term postoperative assessment by Internet-based testing is practical in this patient population. There is a need to assess further the efficacy of SCP, HCA, and other neuroprotective strategies in thoracic aortic surgery by conducting prospective, multicenter, longitudinal studies of long-term neurocognitive outcomes.

We acknowledge the academic contributions of David Erlanger, $\mathrm{PhD}$, and Tanya Kaushik, PsyD, of HeadMinder, Inc., for their guidance in test selection and converting raw neuropsychologic data into standardized scores. We thank Jennifer E. Paress for assistance with data collection.

\section{References}

1. van Dijk D, Keizer AMA, Diephuis JC, Durand C, Vos LJ, Hijman R. Neurocognitive dysfunction after coronary artery bypass surgery: a systematic review. J Thorac Cardiovasc Surg. 2000;120:632-9.

2. Erlanger DM, Kaushik T, Broshek D, Freeman J, Feldman D, Fest J. Development and validation of a web-based screening tool for monitoring cognitive status. J Head Trauma Rehabil. 2002;17:458-76.

3. Ryan SE, Porth LS. A tutorial on the piecewise regression approach applied to bedload transport data: general technical report RMRS-GTR-189. Fort Collins, CO: Department of Agriculture, Forest Service, Rocky Mountain Research Station; 2007.

4. Reich DL, Uysal S, Sliwinski M, Ergin MA, Kahn RA, Konstadt SN, et al. Neuropsychologic outcome after deep hypothermic circulatory arrest in adults. $J$ Thorac Cardiovasc Surg. 1999;117:156-63.

5. Svensson LG, Nadolny EM, Penney DL. Prospective randomized neurocognitive and S-100 study of hypothermic circulatory arrest, retrograde brain perfusion, and antegrade brain perfusion for aortic arch operations. Ann Thorac Surg. 2001;71:1905-12.

6. Slater JP, Guarino T, Stack J, Vinod K, Bustami RT, Brown JM, et al. Cerebral oxygen desaturation predicts cognitive decline and longer hospital stay after cardiac surgery. Ann Thorac Surg. 2009;87:36-45.

7. Knipp SC, Matatko N, Schlamann M, Wilhelm H, Thielmann M, Forsting M, et al. Small ischemic brain lesions after cardiac valve replacement detected by diffusion-weighted magnetic resonance imaging: relation to neurocognitive function. Eur J Cardiothorac Surg. 2005;28:88-96.

8. Harrington DK, Walker AS, Kaukuntla H, Bracewell RM, Clutton-Brock TH, Faroqui M, et al. Selective antegrade cerebral perfusion attenuates brain metabolic deficit in aortic arch surgery: a prospective randomized trial. Circulation. 2004;110(Suppl II):II-231-136.

9. Özatik MA, Küçüker SA, Tülüce H, Sartiaş A, Sener E, Karakaş S, et al. Neurocognitive functions after aortic arch repair with right brachial artery perfusion. Ann Thorac Surg. 2004;78:591-5.

10. Murkin JM, Newman SP, Stump DA, Blumenthal JA. Statement of consensus on assessment of neurobehavioral outcomes after cardiac surgery. Ann Thorac Surg. 1995;59:1289-95. 